\title{
Caracterização morfofisiológica de sementes de diferentes cultivares de soja armazenadas sob condições não controladas
}

\section{Nicolay Wolff Ruppin ${ }^{1 *}$, Lorena Emanuelle da Mata Terra² ${ }^{2}$ Thaíse Ohana Moura Fernandes ${ }^{3}$, Carlos Juliano Brant Albuquerque ${ }^{4}$, Delacyr da Silva Brandão Junior ${ }^{5}$}

\begin{abstract}
Resumo
É de fundamental importância a utilização de sementes de qualidade para obtenção de uniformidade, plantas vigorosas e, por conseguinte boa produtividade. Estando a qualidade de sementes de soja, diretamente relacionada aos atributos físicos, genéticos, sanitários e fisiológicos, objetivou-se com este trabalho, avaliar as características morfofisiológicas e a qualidade de sementes de diferentes cultivares de soja armazenadas durante três meses sob condições não controladas. Foram avaliadas 24 cultivares de soja, em delineamento inteiramente casualizado, quanto a classificação física e morfológica, quanto ao peso de 100 sementes, umidade, teste de peroxidase, germinação, vigor e massa seca da raiz e hipocótilo. Após o armazenamento sob condições não controladas a cultivar NS5959IPRO apresentou maior porcentagem de vigor e germinação (99\%) e maior peso de massa seca de hipocótilo. Das 24 cultivares avaliadas, 14 se enquadram no limite mínimo de qualidade de sementes para comercialização mesmo sob condições de armazenamento não controladas quanto a temperatura e umidade do ar.
\end{abstract}

Palavras-chave: Qualidade. Armazenamento. Características genéticas. Germinação. Glycine max (L.) Merril.

\section{Morphophysiological characterization of seeds of different soybean cultivars stored under uncontrolled conditions}

\begin{abstract}
It is of fundamental importance the use of quality seeds to getting uniformity, vigorous plants and, therefore, good productivity. The quality of soybean seeds, directly related to the physical, genetic, sanitary and physiological attributes, was the objective of this study to evaluate the morphophysiological characteristics and seed quality of different soybean cultivars stored during three months under uncontrolled conditions. Twenty - four soybean cultivars were evaluated in a completely randomized design with respect to the physical and morphological classification of 100 seeds, moisture, peroxidase test, germination, vigor and dry mass of the root and hypocotyl. After storage under uncontrolled conditions the cultivar NS5959IPRO showed a higher percentage of vigor and germination (99\%) and a higher weight of dry mass of hypocotyl. Of the 24 evaluated cultivars, 14 meet the minimum quality limit of seeds for commercialization even under uncontrolled storage conditions regarding temperature and humidity of the air.
\end{abstract}

Keywords: Quality. Storage. Genetic characteristics. Germination. Glycine max (L.) Merril.

\footnotetext{
${ }^{1}$ Universidade Federal de Minas Gerais. Instituto de Ciências Agrárias - MG http://orcid.org/0000-0002-9709-9603

${ }^{2}$ Universidade Federal de Minas Gerais. Instituto de Ciências Agrárias - MG http://orcid.org/0000-0002-3575-8212

${ }^{3}$ Universidade Federal de Minas Gerais. Instituto de Ciências Agrárias - MG http://orcid.org/0000-0001-9565-8865

${ }^{4}$ Universidade Federal de Minas Gerais. Instituto de Ciências Agrárias - MG

http://orcid.org/0000-0003-2244-1336

${ }^{5}$ Universidade Federal de Minas Gerais. Instituto de Ciências Agrárias - MG

http://orcid.org/0000-0001-8967-2847

*Autor para correspondência: nicolaywr@hotmail.com
}

Recebido para publicação em 05 de abril de 2019. Aceito para publicação em 29 de maio de 2019.

e-ISSN: 2447-6218 / ISSN: 2447-6218 / (C) 2009, Universidade Federal de Minas Gerais, Todos os direitos reservados. 


\section{Introdução}

A soja (Glycinemax (L.) Merril) é uma cultura de grande importância para o mercado brasileiro, devido ao seu valor socioeconômico e pela variedade de produtos e subprodutos destinados ao abastecimento interno e externo, sendo amplamente utilizada na indústria química, agroindústria, para alimentação animal e humana (Vinhal-Freitas et al., 2011).

Segundo dados da CONAB (2018), o Brasil é o segundo maior produtor mundial, área plantada de $35.046,5$ milhões de hectares, o que equivale a cerca de $3,4 \%$ a mais que o plantio do ano anterior.

O melhoramento genético vegetal contribuiu de forma significativa para os avanços na produção, tendo possibilitado o desenvolvimento de cultivares adaptáveis às diversas condições edafoclimáticas, bem como o cultivo em regiões de baixas latitudes (Freitas, 2011). Fato que tem favorecido cada vez mais o aumento das produtividades e grandes expansões agrícolas do mercado interno da soja no país.

Contudo a qualidade das sementes é considerada o parâmetro mais importante da produção de soja, pois é através desta que se encontra o material genético responsável pela expressão das características de cada cultivar, portanto, torna-se necessário um controle rigoroso dos fatores que possam interferir na obtenção de sementes com boa qualidade.

Os atributos genéticos, sanitários, físicos, e fisiológicos, são determinantes para a qualidade das sementes. Sendo a qualidade fisiológica de lotes de sementes de soja, facilmente influenciados por características de umidade, tamanho, densidade, doenças, infestação de pragas, danos mecânicos, bem como, condições e tempo de armazenamento (Santos et al., 2007).

O controle da temperatura e umidade são fatores preponderantes para que se mantenha a qualidade das sementes durante o período do armazenamento, de modo a possibilitar a redução das perdas por deterioração. De forma geral o resfriamento dos grãos é suficiente para manter as características fisiológicas, sem que ocorra ataque de pragas e doenças fúngicas. Para Alencar et al. (2009) o padrão básico de comercialização de sementes de soja preconiza umidade de $14 \%$ das sementes para que estas possam ser armazenadas durante 180 dias, sob temperatura em torno de 20 e $30^{\circ} \mathrm{C}$.

A produção de sementes de soja de elevada qualidade é um desafio para o setor sementeiro, principalmente em regiões tropicais e subtropicais do país, sendo necessária a adoção de técnicas especiais, de modo que se possa contornar a influência de fatores abióticos e bióticos. Sendo, portanto, de fundamental importância a escolha de cultivares de alto potencial genético, para que se obtenha altas produtividades em condições adversas de produção (Costa et al., 2003).

Faz-se necessário ainda, estudos sobre as condições de armazenamento das sementes de modo que se possa otimizar tais processos, encontrando condições e material genético ideal para a região em que se deseja utilizar.

Desta forma, objetivou-se com o presente trabalho avaliar a qualidade, através de características genética, física e fisiológica de sementes de 24 cultivares de soja, que foram armazenadas durante três )meses em condições não controladas no Norte de Minas Gerais.

\section{Material e métodos}

O trabalho foi desenvolvido no Laboratório de Análise de Sementes (LAS), localizado no Instituto de Ciências Agrárias, Campus Regional da Universidade Federal de Minas Gerais em Montes Claros. A cidade está situada no Norte do Estado de Minas Gerais a 650 $\mathrm{m}$ de altitude, e nas coordenadas geográficas de $16^{\circ} 43^{\prime} \mathrm{S}$ e $43^{\circ} 53^{\prime} \mathrm{W}$. O clima da região, segundo Alvares et al. (2013), é do tipo Aw, megatérmico, com inverno seco e verão chuvoso. A precipitação média anual é de 1.060 $\mathrm{mm}$ e a temperatura média é de $24,2^{\circ} \mathrm{C}$.

O experimento foi conduzido utilizando sementes de 24 cultivares de sojas, sendo elas M7739IPRO, NA5909RG, NS6909IPRO, 99R09, NS7300IPRO, NS7338IPRO, DM6563RSFIPRO, TMG7062IPRO, RK6813, AS3730IPRO, DS5916IPRO, NS6906IPRO, RK7814, CD2728IPRO, M7110IPRO, CD2817IPRO, CD2720IPRO, AS3610IPRO, BMXPontalIPRO, CD2750IPRO, NS5959IPRO, CD2730IPRO, M8210IPRO e M6210IPRO. As sementes foram obtidas na Fazenda Experimental do Instituto de Ciências Agrárias e foram armazenas quando estas apresentavam umidade de $12 \%$ em sacos de papel, em ambiente não controlado de temperatura e umidade do ar, durante três meses.

A caracterização morfológica das sementes foi determinada medindo-se o comprimento, largura e espessura, com auxílio de um paquímetro digital, utilizando duas repetições de 25 sementes para cada tratamento.

A classificação de peneiras foi realizada utilizando quatro repetições com 25 sementes em peneiras de 9,8 $\mathrm{x} 15,4,5 \mathrm{x} 22$ e $3 \mathrm{~mm}$.

A classificação das sementes quanto à cor do tegumento e hilo, foi realizada de acordo com a metodologia desenvolvida por Munsell (1975).

Foi realizado o teste de peroxidase, retirando-se o tegumento de cada semente colocando-o num tubo de ensaio com dez gotas de solução $0,5 \%$ de guaiacol. 
Caracterização morfofisiológica de sementes de diferentes cultivares de soja armazenadas sob condições não controladas

Após dez minutos, foi adicionado a cada tubo uma gota de água oxigenada 40 volumes, a formação ou não da coloração foi observada após 5 minutos. As cultivares que apresentaram alta atividade da peroxidase no tegumento produziram cor marrom avermelhada, designada como reação positiva, enquanto as cultivares de baixa atividade, não apresentaram alteração na coloração, sendo caracterizado como reação negativa.

A avaliação da qualidade física das sementes foi determinada através da determinação do teor de água, utilizando-se duas repetições com dez sementes para cada cultivar, seguindo as normas prescritas nas RAS (Brasil, 2009). Para tanto, empregou-se o método padrão da estufa a $105 \pm 3^{\circ} \mathrm{C}$ por 24 horas.

O peso de 100 sementes foi determinado utilizando-se duas repetições com 50 sementes para cada tratamento.

A avaliação da qualidade fisiológica foi realizada através do teste padrão de germinação, utilizando-se 100 sementes por cultivar, com quatro repetições. As sementes foram distribuídas em substrato papel germitest, umedecido com água destilada equivalente a 2,5 vezes o seu peso seco e, em seguida foram montados rolos, e acondicionados em um germinador do tipo incubador refrigerador à temperatura constante de $25^{\circ} \mathrm{C}$ e fotoperíodo de 12 horas.

As avaliações de germinação ocorreram no 5ำ e 8ㅇ dia após a instalação do teste. No 5으 dia foi realizada a contagem de plântulas normais emergidas, caracterizadas pela presença de estruturas essenciais intactas (Brasil,

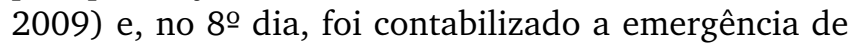

plântulas normais, plântulas anormais, sementes duras e sementes chochas.

Para avaliação do vigor das sementes das diferentes cultivares foram consideradas a porcentagem de plântulas normais emergidas e a porcentagem de germinação ao 5음 DAS (dias após a semeadura).

Após o período de avaliação, as plântulas normais, tiveram o sistema radicular separados da parte aérea, e os cotilédones seccionados, para avaliação da pigmentação antociâninica do hipocótilo e, posteriormente, essas estruturas (sistema radicular e hipocótilo) foram colocada sem sacos de papel e acondicionadas em estufa com circulação forçada de ar, à temperatura de $65^{\circ} \mathrm{C}$, até atingir peso constante. Após esse período as amostras foram pesadas em balança de precisão de 0,001g para obtenção da massa seca (Cavalcante et al., 2012).

Os dados obtidos foram submetidos à análise de variância e comparação de médias pelo teste de Tukey à $5 \%$ de probabilidade, utilizando o programa estatístico software R, versão 3.4.4 (R Core Team, 2018).

\section{Resultados e discussão}

A largura e espessura das sementes não apresentaram diferença estatística entre as diferentes cultivares avaliadas, com valores médios de 6,6018 mm e 5,3681 mm respectivamente. Já o comprimento foi estatisticamente significativo e variou entre 6,905 a $8,214 \mathrm{~mm}$, para as cultivares M6210IPRO e M7739IPRO de menor e maior comprimento respectivamente. Dentre as 24 cultivares avaliadas, todas apresentaram forma alongada (Tabela 1).

Tabela 1 - Comprimento, largura, espessura e forma de sementes de diferentes cultivares de soja, armazenadas sob condições não controladas de temperatura e umidade relativa do ar

\begin{tabular}{clllc}
\hline CULTIVARES & \multicolumn{1}{c}{ COMPRIMENTO } & LARGURA & ESPESSURA & FORMA \\
\hline M7739IPRO & $8.214 \mathrm{a}$ & $6.6062 \mathrm{a}$ & $5.4660 \mathrm{a}$ & Alongada \\
NA5909RG & $8.015 \mathrm{ab}$ & $6.7406 \mathrm{a}$ & $5.5730 \mathrm{a}$ & Alongada \\
NS6909IPRO & $7.991 \mathrm{ab}$ & $6.862 \mathrm{a}$ & $5.6848 \mathrm{a}$ & Alongada \\
99R09 & $7.755 \mathrm{ab}$ & $6.7606 \mathrm{a}$ & $5.1662 \mathrm{a}$ & Alongada \\
NS7300IPRO & $7.688 \mathrm{ab}$ & $6.7258 \mathrm{a}$ & $5.5794 \mathrm{a}$ & Alongada \\
NS7338IPRO & $7.642 \mathrm{ab}$ & $6.6106 \mathrm{a}$ & $5.2580 \mathrm{a}$ & Alongada \\
DM6563RSFIPRO & $7.614 \mathrm{ab}$ & $6.8104 \mathrm{a}$ & $5.5492 \mathrm{a}$ & Alongada \\
TMG7062IPRO & $7.603 \mathrm{ab}$ & $6.9500 \mathrm{a}$ & $5.7348 \mathrm{a}$ & Alongada \\
RK6813 & $7.586 \mathrm{ab}$ & $6.5870 \mathrm{a}$ & $5.4168 \mathrm{a}$ & Alongada \\
AS3730IPRO & $7.543 \mathrm{ab}$ & $6.7254 \mathrm{a}$ & $5.4036 \mathrm{a}$ & Alongada \\
DS5916IPRO & $7.498 \mathrm{ab}$ & $6.5482 \mathrm{a}$ & $5.5050 \mathrm{a}$ & Alongada \\
\hline
\end{tabular}


Ruppin N. W. et al.

\begin{tabular}{ccccc}
\hline CULTIVARES & COMPRIMENTO & LARGURA & ESPESSURA & FORMA \\
\hline NS6906IPRO & $7.484 \mathrm{ab}$ & $6.9958 \mathrm{a}$ & $5.4096 \mathrm{a}$ & Alongada \\
RK7814 & $7.440 \mathrm{ab}$ & $6.5308 \mathrm{a}$ & $5.3650 \mathrm{a}$ & Alongada \\
CD2728IPRO & $7.427 \mathrm{ab}$ & $6.7542 \mathrm{a}$ & $5.5132 \mathrm{a}$ & Alongada \\
M7110IPRO & $7.388 \mathrm{ab}$ & $6.6832 \mathrm{a}$ & $5.1060 \mathrm{a}$ & Alongada \\
CD2817IPRO & $7.358 \mathrm{ab}$ & $5.9852 \mathrm{a}$ & $4.8544 \mathrm{a}$ & Alongada \\
CD2720IPRO & $7.344 \mathrm{ab}$ & $6.5064 \mathrm{a}$ & $5.1460 \mathrm{a}$ & Alongada \\
AS3610IPRO & $7.251 \mathrm{ab}$ & $6.4502 \mathrm{a}$ & $5.3630 \mathrm{a}$ & Alongada \\
BMXPontaIPRO & $7.241 \mathrm{ab}$ & $6.4393 \mathrm{a}$ & $5.2886 \mathrm{a}$ & Alongada \\
CD2750IPRO & $7.106 \mathrm{ab}$ & $6.4302 \mathrm{a}$ & $5.1888 \mathrm{a}$ & Alongada \\
NS5959IPRO & $7.100 \mathrm{ab}$ & $6.7202 \mathrm{a}$ & $5.6622 \mathrm{a}$ & Alongada \\
CD2730IPRO & $7.045 \mathrm{ab}$ & $6.4788 \mathrm{a}$ & $5.2810 \mathrm{a}$ & Alongada \\
M8210IPRO & $6.955 \mathrm{~b}$ & $6.3392 \mathrm{a}$ & $5.2524 \mathrm{a}$ & Alongada \\
M6210IPRO & $6.905 \mathrm{~b}$ & $6.2018 \mathrm{a}$ & $5.0680 \mathrm{a}$ & Alongada \\
\hline CV & $4.08 \%$ & $4.8 \%$ & $5.61 \%$ & \\
\hline
\end{tabular}

Médias seguidas de mesma letra na coluna não diferem entre si pelo teste Tukey a 5\% do nível de significância.

De acordo com o teste de peneiras $90 \%$ das sementes de todas as cultivares utilizadas foram classificadas com 4,5 x $22 \mathrm{~mm}$. O tamanho das sementes representa característica importante de qualidade, uma vez que são diretamente relacionados ao vigor das plantas, sendo que sementes de maior tamanho tendem a apresentar maior quantidade de reservas e embriões mais bem formados (Pádua et al., 2010), bem como a padronização do tamanho e forma do lote, influenciam de forma direta na mecanização durante o plantio.
Para Soares et al. (2013), "além de estar diretamente relacionado ao vigor de plântulas, o maior tamanho das sementes de soja possui influência genética na produção de sementes maiores e mais pesadas".

Quanto a coloração do hilo as cultivares apresentaram variação entre as cores, amarela, marrom e cinza. A coloração do hipocótilo foi presente em apenas 2, das 24 cultivares avaliadas. A coloração do tegumento foi variável entre as cores marrom e amarelo de acordo com a cultivar (Tabela 2).

Tabela 2 - Coloração do hilo, tegumento e hipocótilo de diferentes cultivares de soja, de acordo com a carta de cores de Munsell

\begin{tabular}{cccc}
\hline CULTIVAR & HILO & TEGUMENTO & HIPOCÓTILO \\
\hline 99R09 & 10YR 4/2 darkgrayishbrown & $2.5 Y$ 8/6 yellow & ausente \\
AS3610IPRO & 10YR 7/6 yellow & 2.5Y 8/4 paleyellow & ausente \\
AS3730IPRO & 10YR 6/6 brownsihyellow & 2.5Y 8/4 paleyellow & ausente \\
BMXPontaIPRO & 10YR 5/6 yellowishbrown & $2.5 Y$ 8/6 yellow & ausente \\
CD2720IPRO & 10YR 4/2 darkgrayishbrown & 10YR 7/4 verypalebrown & presente \\
CD2728IPRO & 10YR 4/3 darkbrown & 2.5Y 8/4 paleyellow & ausente \\
D2730IPRO & 10YR 4/2 darkgrayishbrown & 10YR 8/4 verypaleyellow & ausente \\
CD2750IPRO & 10YR 5/2 grayishbrown & 10YR 7/4 verypalebrown & ausente \\
CD2817IPRO & 10YR 6/6 brownsihyellow & $2.5 Y$ 7/6 yellow & ausente \\
DM6563RSFIPRO & 10YR 6/6 brownsihyellow & 10YR 8/4 verypaleyellow & ausente \\
DS5916IPRO & 10YR 6/6 brownsihyellow & $2.5 Y$ 8/6 yellow & presente \\
\hline
\end{tabular}


Caracterização morfofisiológica de sementes de diferentes cultivares de soja armazenadas sob condições não controladas

\begin{tabular}{cc}
\hline CULTIVAR & HILO \\
\hline M6210IPRO & 10 YR 5/4 darkyeloowishbrow \\
M7110IPRO & 10YR 5/6 yellowishbrown \\
M7739IPRO & 10YR 3/1 verydarkgray \\
M8210IPRO & 10YR 3/1 verydarkgray \\
NA5909RG & 10YR 4/3 darkbrown \\
NS5959IPRO & 10 YR 4/4 darkyeloowishbrow \\
NS6906IPRO & 2.5Y 7/6 yellow \\
NS6909IPRO & 10YR 4/3 darkbrown \\
NS7300IPRO & 10YR 4/3 brown \\
NS7338IPRO & 10YR 4/4 darkyellowihbrown \\
TMG7062IPRO & 10YR 7/6 yellow \\
RK6813 & 10YR 6/6 brownsihyellow \\
RK7814 & 10YR 3/1 verydarkgray
\end{tabular}

\begin{tabular}{cc}
\hline CULTIVAR & REAÇÃO \\
\hline DS5916IPRO & + \\
M6210IPRO & + \\
M7110IPRO & - \\
M7739IPRO & - \\
M8210IPRO & + \\
NA5909RG & + \\
NS5959IPRO & - \\
NS6906IPRO & + \\
NS6909IPRO & - \\
NS7300IPRO & + \\
NS7338IPRO & + \\
TMG7062IPRO & - \\
RK6813 & + \\
RK7814 & + \\
\hline
\end{tabular}

+ Reação positiva da peroxidase

- Reação negativa da peroxidase

$+\mathrm{e}-$ Reação positiva e negativa da peroxidase

A cultivar AS3730IPRO apresentou reação positiva e negativa para a atividade da peroxidase. Tal resultado implica uma provável mistura varietal ou ocorrência de segregação, uma vez que esta resposta é considerada atípica, por se tratar de uma característica designada geneticamente (Silva e Bonacina, 2014).

De acordo com as análises estatísticas $(\mathrm{P}<0,05)$ as variáveis peso de 100 sementes (g) e umidade (\%), 
Ruppin N. W. et al.

não foram consideradas significativamente diferentes para as cultivares testadas, apresentando média de 8,22 g e $6,91 \%$ respectivamente.

Como esperado, ouve uma redução significativa no teor de água das sementes de 12 para 6,91\%, uma vez que, o aumento da temperatura interfere de forma direta na umidade das sementes e, por conseguinte na redução do seu peso, no entanto, por se tratar de uma semente ortodoxa, ou seja, considerada tolerante à dessecação, as sementes de soja podem apresentar redução do teor de umidade até $5 \%$ sem que se tenha perdas de viabilidade. Por outro lado, as sementes de soja são consideradas de vida curta sendo necessários cuidados, quanto à temperatura e umidade no armazenamento, uma vez que condições desfavoráveis podem provocar sua deterioração (Ellis et al., 1991).

As sementes sofrem perdas consideráveis de umidade à medida em que se aumenta o tempo de armazenamento e em decorrência da embalagem utilizada para o acondicionamento das sementes, uma vez que, as embalagens de papel são permeáveis, tendem a sofrer influência da atmosfera permitindo troca de vapor de água com o ambiente no qual está armazenado, o que reduz o teor de água das sementes (Smaniotto et al., 2014). Para Alencar et al. (2009) "a umidade de até 11\% é ideal para o armazenamento de sementes de soja, para manter a longevidade e a qualidade das sementes".

A germinação total apresentou diferença significativa $(P<0,05)$, apresentando maior germinação total para a cultivar NS5959IPRO, referente a 99\% enquanto as cultivares M6210IPRO e 99R09 apresentaram menor atributo de qualidade, representados por 48 e 39\% de germinação (Tabela 4).

O vigor, expresso pela contagem de plântulas normais e pela germinação ao 5음 DAS foi estatisticamente diferente para as 24 cultivares avaliadas $(\mathrm{P}<0,05)$, obtendo-se maior porcentagem de plântulas normais para a cultivarNS5959IPRO, apresentando 98\% e menor para as cultivares M6210IPRO e 99R09, que apresentaram 40 e $21 \%$ respectivamente. Quanto a germinação na primeira contagem a cultivar CD2750IPRO apresentou-se a mais vigorosa com $89 \%$ de germinação e menor para a cultivar M7739IPRO com 8\% de germinação (Tabela 4).

Tabela 4 - Porcentagem de Germinação em primeira contagem (5o DAS), Germinação total e Plântulas normais de diferentes cultivares de soja armazenadas sob condições não controladas de temperatura e umidade relativa do ar

\begin{tabular}{cccc}
\hline CULTIVAR & $\begin{array}{c}\text { GERMINAÇÃO } \\
\text { 1a CONTAGEM (\%) }\end{array}$ & $\begin{array}{c}\text { GERMINAÇÃO } \\
\text { TOTAL (\%) }\end{array}$ & $\begin{array}{c}\text { PLÂNTULAS NORMAIS } \\
(\%)\end{array}$ \\
\hline CD2750IPRO & $89 \mathrm{a}$ & $96 \mathrm{ab}$ & $93 \mathrm{ab}$ \\
AS3730IPRO & $86 \mathrm{ab}$ & $95 \mathrm{ab}$ & $92 \mathrm{ab}$ \\
NS5959IPRO & $84 \mathrm{abc}$ & $99 \mathrm{a}$ & $98 \mathrm{a}$ \\
NA5909RG & $79 \mathrm{abc}$ & $95 \mathrm{ab}$ & $92 \mathrm{ab}$ \\
NS6909IPRO & $77 \mathrm{abc}$ & $90 \mathrm{~b}$ & $84 \mathrm{ab}$ \\
NS6906IPRO & $75 \mathrm{abc}$ & $86 \mathrm{abc}$ & $85 \mathrm{ab}$ \\
CD2720IPRO & $73 \mathrm{abcd}$ & $90 \mathrm{~b}$ & $86 \mathrm{ab}$ \\
DS5916IPRO & $72 \mathrm{abcd}$ & $95 \mathrm{ab}$ & $92 \mathrm{ab}$ \\
CD2730IPRO & $71 \mathrm{abcd}$ & $92 \mathrm{ab}$ & $86 \mathrm{ab}$ \\
TMG7062IPRO & $70 \mathrm{abcd}$ & $94 \mathrm{ab}$ & $88 \mathrm{ab}$ \\
BMXPontaIPRO & $68 \mathrm{abcd}$ & $97 \mathrm{ab}$ & $95 \mathrm{ab}$ \\
NS7300IPRO & $67 \mathrm{abcd}$ & $83 \mathrm{bc}$ & $79 \mathrm{ab}$ \\
RK6813 & $65 \mathrm{abcd}$ & $89 \mathrm{~b}$ & $83 \mathrm{ab}$ \\
DM6563RSFIPRO & $63 \mathrm{abcd}$ & $87 \mathrm{~b}$ & $82 \mathrm{ab}$ \\
AS3610IPRO & $62 \mathrm{abcd}$ & $83 \mathrm{bc}$ & $76 \mathrm{ab}$ \\
RK7814 & $58 \mathrm{abcd}$ & $82 \mathrm{bc}$ & $76 \mathrm{ab}$ \\
CD2728IPRO & $57 \mathrm{abcde}$ & $80 \mathrm{bc}$ & $73 \mathrm{c}$ \\
\hline
\end{tabular}


Caracterização morfofisiológica de sementes de diferentes cultivares de soja armazenadas sob condições não controladas

\begin{tabular}{cccc}
\hline CULTIVAR & $\begin{array}{c}\text { GERMINAÇÃO } \\
\text { 1 a CONTAGEM (\%) }\end{array}$ & $\begin{array}{c}\text { GERMINAÇÃo } \\
\text { TOTAL (\%) }\end{array}$ & $\begin{array}{c}\text { PLÂNTULAS NORMAIS } \\
\text { (\%) }\end{array}$ \\
\hline M8210IPRO & $52 \mathrm{bcde}$ & $84 \mathrm{bc}$ & $74 \mathrm{c}$ \\
M7110IPRO & $38 \mathrm{cde}$ & $57 \mathrm{~d}$ & $51 \mathrm{~d}$ \\
NS7338IPRO & $30 \mathrm{de}$ & $56 \mathrm{~d}$ & $45 \mathrm{~d}$ \\
CD2817IPRO & $23 \mathrm{ef}$ & $73 \mathrm{c}$ & $55 \mathrm{~d}$ \\
M6210IPRO & $20 \mathrm{f}$ & $48 \mathrm{e}$ & $40 \mathrm{~d}$ \\
99R09 & $13 \mathrm{fg}$ & $39 \mathrm{f}$ & $21 \mathrm{e}$ \\
M7739IPRO & $8 f g$ & $93 \mathrm{ab}$ & $89 \mathrm{ab}$ \\
\hline CV & $14,11 \%$ & $8,32 \%$ & $9,46 \%$ \\
\hline
\end{tabular}

Médias seguidas de mesma letra na coluna não diferem entre si pelo teste Tukey a 5\% do nível de significância.

Das 24 cultivares analisadas, 14 se enquadram no limite germinativo para comercialização de sementes de soja ( $\geq 80 \%$ ), o que demonstra o desempenho e a qualidade fisiológica das diferentes cultivares frente a tolerância ao armazenamento para as condições ambientais do Norte de Minas Gerais (Mapa, 2013).

Quanto ao vigor 14 das 24 cultivares avaliadas apresentaram acima de $80 \%$ de plântulas normais emergidas e apenas 3 apresentaram acima de $80 \%$ de germinação ao 5o DAS.

O alto vigor é expresso em sementes por proporcionar maior velocidade nos processos metabólicos, favorecendo assim a emergência das plântulas, através da rápida emissão das estruturas primárias e maior taxa de crescimento (Munizzi et al., 2010).

O tempo de armazenamento em embalagens permeáveis e sob condições de temperatura e umidade do ar não controlados, podem interferir no vigor e germinação das sementes, podendo causar sua deterioração (Smaniotto et al., 2013), tais aspectos no entanto, são características determinadas geneticamente, demonstrando que cultivares mais resistentes podem apresentar-se viáveis diante de condições de estresse.

A massa seca do hipocótilo e raiz também apresentou diferença significativa entre os resultados das cultivares testadas $(\mathrm{P}<0,05)$, variando de 0,511 a $0,067 \mathrm{~g}$ para as cultivares NS5959IPRO e 99R09 respectivamente (Tabela 5).
Tabela 5 - Peso seco da raiz e hipocótilo das plântulas normais de diferentes cultivares de soja, armazenadas sob condições não controladas de temperatura e umidade relativa do ar

\begin{tabular}{cc}
\hline CULTIVAR & $\begin{array}{c}\text { PESO SECO DA RAIZ E } \\
\text { HIPOCÓTILO }(\mathrm{G})\end{array}$ \\
\hline NS5959IPRO & $0,512 \mathrm{a}$ \\
BMXPontaIPRO & $0,490 \mathrm{ab}$ \\
M7739IPRO & $0,465 \mathrm{ab}$ \\
CD2750IPRO & $0,455 \mathrm{abc}$ \\
AS3730IPRO & $0,454 \mathrm{abc}$ \\
CD2720IPRO & $0,437 \mathrm{abc}$ \\
NA5909RG & $0,420 \mathrm{abc}$ \\
DS5916IPRO & $0,402 \mathrm{abcd}$ \\
TMG7062IPRO & $0,384 \mathrm{abcd}$ \\
DM6563RSFIPRO & $0,384 \mathrm{abcd}$ \\
CD2728IPRO & $0,377 \mathrm{abcd}$ \\
NS7300IPRO & $0,369 \mathrm{abcd}$ \\
NS6906IPRO & $0,361 \mathrm{bcd}$ \\
NS6909IPRO & $0,360 \mathrm{bcd}$ \\
CD2730IPRO & $0,358 \mathrm{bcd}$ \\
RK7814 & $0,348 \mathrm{bcd}$ \\
RK6813 & $0,348 \mathrm{bcd}$ \\
AS3610IPRO & $0,321 \mathrm{~cd}$ \\
M8210IPRO & $0,272 \mathrm{de}$ \\
M7110IPRO & $0,176 \mathrm{ef}$ \\
CD2817IPRO & $0,158 \mathrm{ef}$ \\
\hline
\end{tabular}




\begin{tabular}{cc}
\hline & \\
\hline CULTIVAR & $\begin{array}{c}\text { PESO SECO DA RAIZ E } \\
\text { HIPOCÓTILO (G) }\end{array}$ \\
NS7338IPRO & $0,136 \mathrm{ef}$ \\
M6210IPRO & $0,134 \mathrm{ef}$ \\
$99 \mathrm{R} 09$ & $0,067 \mathrm{f}$ \\
\hline CV & $33,81 \%$
\end{tabular}

Médias seguidas de mesma letra na coluna não diferem entre si pelo teste Tukey a 5\% do nível de significância.

De acordo com Santos et al. (2017), sementes mais vigorosas tendem a originar plântulas mais desen- volvidas e com maior teor de massa, o que confere a estas melhores condições de adaptação e estabelecimento em campo.

\section{Conclusões}

Das 24 cultivares avaliadas, 14 não apresentam redução da qualidade fisiológica, durante o armazenamento sob condições não controladas de temperatura e umidade do ar no Norte de Minas Gerias, uma vez que, a porcentagem de germinação se enquadra dentro dos limites exigidos pelo MAPA para a comercialização de sementes.

\section{Referências}

Alencar, E. R.; Faroni, L. R. D.; Lacerda Filho, A. F.; Peternelli, L. A.; Costa, A. R. 2009. Qualidade dos grãos de soja armazenados em diferentes condições. Revista Brasileira de Engenharia Agrícola e Ambiental, 13: 606-6013. Doi: http://doi.org/10.1590/S1415-43662009000500014.

Alvares, C. A.; Stape, J. L.; Sentelhas, P. C.; Gonçalves, J. L. de M.; Sparovek, G. 2013. Köppen's climatec classification map for Brazil. Meteorologische Zeitschrift, 22: 711-728. Doi: http://dx.doi. org/10.1127/0941-2948/2013/0507.

Cavalcante, V. S.; Santos, V. R.; Santos Neto, A. L.; Santos, M. A. L.; Santos, C. G.; Costa, L. C. 2012. Biomassa e extração de nutrientes por planta de cobertura. Revista Brasileira de Engenharia agrícola e Ambiental, 16: 521-528. Doi: http://dx.doi.org/10.1590/S141543662012000500008 .

Costa. N. P.; Mesquita, C. M.; Maurina, A. C.; França-Neto, J. B.; KrzyzanowskI, F. C.; Henning, A. A. 2003. Qualidade fisiológica, física e sanitária de sementes de soja produzidas no Brasil. Revista Brasileira de Sementes, 25: 128-132. Doi: http://dx.doi.org/10.1590/S010131222003000100020 .

Ellis, R. H.; Hong, T. D.; Roberts, E. H.; Soetisna, U. 1991. Seed storage behaviour in Elaeis guineenses. Journal of Experimental Botany, 1: 99104. Doi: https://dx.doi.org/10.1017/S0960258500000726.

Freitas, M. C. M. 2011. A cultura da soja no Brasil: o crescimento da produção brasileira e o surgimento de uma nova fronteira agrícola. Enciclopédia Biosfera, Centro Científico Conhecer, 7: 1-12.

Ministério da Agricultura, Pecuária e Abastecimento. 2009. Regras para análise de sementes / Ministério da Agricultura, Pecuária e Abastecimento - MAPA. Secretaria de Defesa Agropecuária. Brasília.

Ministério da Agricultura, Pecuária e Abastecimento. 2009. Instrução normativa no 45, de 17 de setembro de 2013 [MAPA]. Disponível em <http://www.agricultura.gov.br/assuntos/insumos-agropecuarios/ insumos-agricolas/sementes-e-mudas/publicacoes-sementes-e-mudas > Acesso em 14 jun de 2019.

Munizzi, A.; Braccini.; A. L.; Rangel, M. A. S; Scapim; C. A; Albrecht, L. P. 2010. Qualidade de sementes de quatro cultivares de soja, colhidas em dois locais no estado de Mato Grosso do Sul. Revista Brasileira de Sementes, 32: 176-185. Doi: http://dx.doi.org/10.1590/S010131222010000100020 .
Munsell, C. 1975. Munsell Soil Color Charts: Munsell Color. Baltimore, Maryland.

Pádua, G. P.; Zito, R. K.; Arantes, N. E.; França-Neto, J. B. 2010. Influência do tamanho da semente na qualidade fisiológica e na produtividade da cultura da soja. Revista Brasileira de Sementes, 32: 009-016. Doi: http://dx.doi.org/10.1590/S0101-31222010000300001.

Rabel, M.; Vieira, E. S. N.; Lana, U. G. P; Paiva, E.; Sehnem, M. A. S.; Schuster, I. 2010. Marcadores moleculares microssatélites na avaliação de sementes de soja com variação na coloração do hilo. Revista Brasileira de Sementes, 32: 019-025. Doi: http://dx.doi.org/10.1590/S010131222010000200002 .

Team, R. C. 2018. R Foundation for Statistical Computing. R: A language and environment for statistical computing. Vienna: R Foundation for Statistical Computing.

Santos, A. S.; Souza, E. M.; Féboli, A.; Nogueira, D. C. 2017. Teste de vigor em sementes de três cultivares de soja. Revista Conexão Eletrônica, 14: 1-12.

Santos, E. L.; Póla, J. N.; Barros, A. S. R.; Prete, C. E. C. 2007. Qualidade fisiológica e composição química das sementes de soja com variação na cor do tegumento. Revista Brasileira de Sementes, 29: 20-26. Doi: http://dx.doi.org/10.1590/S0101-31222007000100003.

Silva, R. J. L.; Bonacina, R. 2014. Caracterização de cultivares de soja por meio de testes da peroxidase utilizando o método do tegumento e da semente inteira. Revista Eletrônica da Faculdade de Ciências Exatas e da Terra, 3: 19-27.

Smaniotto, T. A. S.; Resende, O.; Marçal, K. A. F.; Oliveira, D. E. C.; Simon, G. A. 2014. Qualidade fisiológica de sementes de soja armazenadas em diferentes condições. Revista Brasileira de Engenharia Agrícola e Ambiental, 18: 446-453. Doi: http://dx.doi.org/10.1590/S141543662014000400013 .

Soares, M. M.; Oliveira, G. L.; Soriano, P. E.; Sekita, M. C.; Sediyama, T. 2013. Performance os soybean plantas as function of seed size: II. Nutricional stress. Journal of Seed Science, 35: 419-427. Doi: http:// dx.doi.org/10.1590/S2317-15372013000400002.

Vinhal-Freitas, I. C.; Nunes Júnior, J. E. G.; Segundo, J. P.; Vilarinho, M. S. 2011. Germinação e vigor de sementes de soja classificadas em diferentes tamanhos. Agropecuária Técnica, 32: 108-114. DOI: https:// doi.org/10.25066/agrotec.v32i1.9567. 